\title{
Tendencias en los temas de los conceptos del Consejo Técnico de la Contaduría Pública como organismo normalizador técnico contable en Colombia
}

Trends in issues of the concepts of the Consejo Técnico de la Contaduría Pública as a technical accounting standardizing body in Colombia

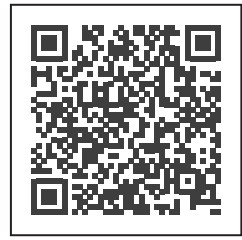

Palabras claves:

concepto, contabilidad, GESTIO, organismo normalizador, tendencia.

Artículo de investigación

Fecha de recepción:

28/08/2020

Fecha de aprobación:

27/05/2021

Fecha de publicación

25/06/2021

Creative Commons

Reconocimiento-NoComercial-SinObraDerivada 4.0 Internacional

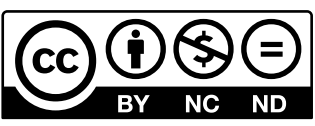

Rodolfo Antonio Munevar-Moreno';

Angie Viviana Barón-Vivas²; Yerly Andrea Galindo-Rodríguez ${ }^{3}$

\section{Resumen}

Las fuentes de información documental que emiten organismos normalizadores como el Consejo Técnico de la Contaduría Pública (CTCP) son documentos que aportan la evolución y el desarrollo de una institución y ameritan la revisión desde varios enfoques en la investigación. Un ejemplo es los conceptos que se originan a partir de las consultas realizadas por personas naturales y jurídicas tanto del sector privado como del sector público. El artículo tiene como propósito establecer las tendencias de temas de los conceptos emitidos y publicados por parte del

1 Contador público, especialista en Revisoría Fiscal y Contraloría. Tesista para optar al título de magíster en Historia. Estudiante del doctorado en Ciencias Contables. Docente investigador líder, semillero de investigación Otras Realidades de la Contabilidad, Grupo RE-DES, programa de Contaduría Pública, Fundación Universitaria Juan de Castellanos, Colombia. rodolfo. munevar@gmail.com, ORCID: https://orcid.org/0000-0003-0617-6819

2 Contadora pública. Joven investigador, semillero de investigación Otras Realidades de la Contabilidad, grupo de investigación RE-DES, programa de Contaduría Pública, Fundación Universitaria Juan de Castellanos, Colombia. vivianabaronvivas@gmail.com ORCID: https://orcid.org/0000-0001-6727-8531

3 Contadora pública. Joven investigador, semillero de investigación Otras Realidades de la Contabilidad, grupo de investigación RE-DES, programa de Contaduría Pública, Fundación Universitaria Juan de Castellanos, Colombia. andreagalindo2710@gmail.com ORCID: https://orcid.org/0000-0003-2097-4593 


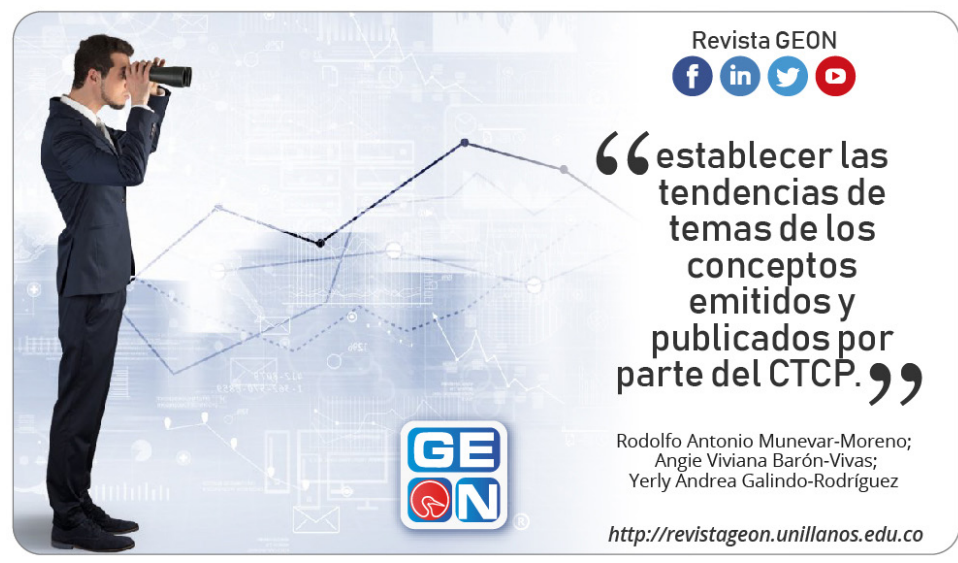

CTCP en el período comprendido entre el año 1992 y el año 2018. En este avance se revisó el contenido de 6578 conceptos de 7191 que están publicados en la página del CTCP, estableciendo categorías relacionadas con los elementos de los estados financieros, el tipo de persona que realiza la consulta y el sector al que pertenece, utilizando la frecuencia, herramienta de la estadística descriptiva en los valores encontrados de las categorías definidas.

Como conclusión principal, se encuentra un punto de inflexión en el año 2009, que refiere a un aumento de emisión y publicación de conceptos por parte del CTCP dado posiblemente por el cambio de modelo contable en Colombia para ese mismo año. La contribución del artículo es un avance y propuesta para trabajar con fuentes documentales emitidas por instituciones públicas de la profesión, para este caso, con un enfoque descriptivo, pero que pueda abarcarse desde otros enfoques y metodologías de investigación.

Cómo citar este artículo/ Toreference this article:

Munevar Moreno, R. A., Barón-Vivas, A. V., \& Galindo-Rodríguez, Y. A. (2021). Tendencias en los temas de los conceptos del Consejo Técnico de la Contaduría Pública como organismo normalizador técnico contable en Colombia. Revista GEON (Gestión, Organizaciones Y Negocios), 8(1), e-227. https://doi. org/10.22579/23463910.227
Palabras claves: concepto, contabilidad, GESTIO, organismo normalizador, tendencia.

Códigos JEL: M41Contabilidad, M48 Política y regulación del gobierno

\section{Abstract}

The sources of documentary information issued by standardizing bodies such as the Consejo Técnico 
Cómo citar este artículo / Toreference this article:

Munevar Moreno, R. A., Barón-Vivas, A. V., \& Galindo-Rodríguez, Y. A. (2021). Tendencias en los temas de los conceptos del Consejo Técnico de la Contaduría Pública como organismo normalizador técnico contable en Colombia. Revista GEON (Gestión, Organizaciones Y Negocios), 8(1), e-227. https://doi. org/10.22579/23463910.227 de la Contaduría Pública (CTCP) are documents that provide the evolution and development of an institution and merit review from various research approaches; an example, are the Concepts that originate from the consultations made by natural and legal persons from both the private and public sectors. The purpose of the article is to establish the trends in the topics of the concepts issued and published by the CTCP in the period between 1992 and 2018. In this advance, the content of 6,578 concepts out of 7,191 that are published in the page of the CTCP, establishing categories related to the elements of the financial statements, the type of person making the query and the sector to which it belongs, using the frequency, tool, of descriptive statistics on the values found in the defined categories.

The main conclusion is a turning point in 2009, which refers to an increase in the issuance and publication of concepts by the CTCP possibly due to the change in the accounting model in Colombia for that same year; the contribution of the article is an advance and proposal to work with documentary sources issued by public institutions of the profession, in this case with a descriptive approach, but which can be covered from other research approaches and methodologies.

Keywords: Accounting; Concept; GESTIO; Normalizing body; Trend.

JEL codes: M41 Accounting, M48 Government Policy and Regulation

\section{Introducción}

El Consejo Técnico de la Contaduría Pública (CTCP) es un organismo permanente de normalización técnica de normas contables, de información financiera y de aseguramiento de la información, adscrito al Ministerio de Comercio, Industria y Turis- mo (MinCIT). Algunas de las funciones más relevantes del CTCP son: realizar propuestas al MinCIT para la expedición de principios, normas, guías e interpretaciones en materia contable; revisar la concordancia entre las normas expedidas para el proceso de convergencia y emitir conceptos resolviendo las diferentes inquietudes 
que resultan del desarrollo de la profesión contable; esta última es una de las más requeridas por terceros, profesionales y empresas.

Por lo anterior, el CTCP se convierte en el organismo de normalización técnica contable que, aunque no emite la normatividad directamente en el país, sí establece un trabajo previo para realizar las propuestas y a la vez desarrolla un proceso posterior de análisis, entre otras, de las dudas que surgen en el proceso de la implementación del modelo NIIF en Colombia. El CTCP se ha venido convirtiendo en un organismo de relevancia y dentro de sus aportes deja evidencia a través de documentos como los conceptos, aspectos que pueden ayudar a comprender no solo las políticas nacionales en materia de contabilidad, sino también su propia evolución.

Aunque no se han encontrado estudios donde se utilicen los conceptos como fuentes documentales de investigación, este artículo tiene como objetivo establecer las tendencias de temas de los conceptos emitidos y publicados por parte del CTCP en el período comprendido entre el año 1992 y el año 2018. De manera descriptiva, este texto es el primer avance de investigación; lo anterior hace parte de un proyecto de investigación más amplio que tiene como objetivo analizar la evolución del CTCP como organismo e institución de normalización técnica contable.

La importancia del tema de investigación es el interés general para la profesión contable en Colombia. A los terceros, profesionales, empresas y otras instituciones, en sus actividades ordinarias, les surgen dudas y dificultades en la aplicación no solo de la normatividad contable, sino también de la de otras áreas relacionadas con el profesional de la contaduría, como la tributaria (Almeida Espinosa, \& Rojas Prada, 2018)o comercial, por tomar dos ejemplos, las cuales son consultadas al CTCP, organismo e institución que desde la ley 1314 y sus normas reglamentarias le han conferido dentro de sus funciones.

\section{Contexto teórico}

\section{Organismo e institución}

Un primer aspecto que debe tenerse en cuenta para el texto es el tomar al CTCP no solo como un organismo, sino como una institución, la cual, además de cumplir unas funciones dentro del ámbito colombiano en materia contable, se establece como un actor antes y durante el proceso de convergencia al modelo de NIIF iniciado por la ley 1314 de 2009, "[...] delegando al Consejo Técnico de la Contaduría Pública (CTCP) como ente normalizador" (Fuentes et al., 2011, p. 11).

Lo mencionado anteriormente no es un tema aislado, sino que se configura dentro de una política pública para cumplir parámetros en organismos multilaterales. Un autor como Martínez (2011) menciona que "[...] dentro del panorama globalizador, se ha venido produciendo el proceso de normalización contable, que no es otra cosa que la implantación de 
estereotipos contables impulsados por organismos como el AICPA, IFAC y IASC"(p. 25). Lo anterior se cerca a lo que propone el mismo autor sobre el proceso de globalización en materia contable, en relación con "[...] la preocupación por expandir el aire regulativo de la actividad contable hacia los países latinoamericanos"(Martínez, 2000, p. 141).

Las posturas sobre el proceso de convergencia hacia las NIIF fueron diversas y presentaron en su momento posiciones extremas de pesimismo, para unos, o como una oportunidad para proponer cambios no solo en aspectos contables, sino de la profesión, para otros; por ejemplo, Sierra (2002), en su momento, afirmó:

La regulación contable emitida en Colombia debe consultar la situación real del medio económico, político, cultural, social y jurídico. Copiar o adoptar normas de otro país, sin tener en cuenta lo anterior, son actos que deben evitarse. El estado actual de la regulación contable en nuestro país ha despertado el interés por el estudio y definición de las bases teóricas que soportan dicha regulación y una preocupación profesional y académica por el desarrollo de la teoría e investigación contables en el medio nacional. (p. 103)

Sin embargo, un aporte de Sierra (2002) para la construcción de lo teórico es la argumentación de "instituciones consultivas" conformadas por los mismos profesionales de la contaduría. Este señala claramente que:
[...] la creación de instituciones consultivas como el consejo permanente, reviste especial importancia en la medida que gran parte de su participación en el proceso de expedición, modificación y compresión regulativa, recae en quienes participan de manera activa a su interior; la profesión juega un importante papel en este contexto. (p. 103)

Lo que tal vez no definía era su origen, en cuanto a lo público o privado, debate que también se da en diferentes países al ser los organismos emisores de carácter privado, aunque adoptados por los países claramente con un enfoque público.

Otro aspecto que debe tenerse en cuenta dentro de la institucionalidad son los sistemas jurídicos establecidos y usados en este contexto. De acuerdo con Mila y Yáñez (2020), se destacan dos sistemas jurídicos básicos: "a) continental o de derecho escrito (Written Law) y b) anglosajón o de derecho consuetudinario (Common Law)" (p. 76). El primero refiere a un sistema de derecho normativo donde la norma establece los parámetros y el segundo refiere al derecho consuetudinario, con una influencia anglosajona por la que la práctica y lo específico se sobreponen a las normas de carácter general. Para el caso colombiano, es claro que está sostenido en un sistema de derecho normativo, pero construye su modelo contable a partir del año 2009 adoptando normas que son construidas sobre un sistema de derecho consuetudinario, donde está inmerso el 
CTCP como organismo normalizador, aunque no regulador, la segunda facultad del legislador o del ejecutivo en su función reglamentaria.

La institucionalidad es más entendida dentro de Estado o gobierno y se relaciona con la autoridad, la legalidad y las garantías que pueda dar un proceso o actividad (Fuentes et al., 2011, p. 11). Para el caso colombiano, fue a un organismo público, el CTCP, al cual se le delegaron las funciones de organismo normalizador técnico contable, de acuerdo con Arango y Torres (2014):

[...] como autoridad de normalización técnica propone los proyectos de las normas de contabilidad e información financiera y de aseguramiento de la información y coordina el proceso previo de su emisión; adicionalmente, es el órgano asesor y consultor de normatividad contable catalogado como el máximo órgano de dirección y orientación técnica científica de la profesión. (p. 135)

Sin embargo, en otros países donde se hicieron procesos similares al colombiano, se establecieron organismos de emisión privados más que de asesores, consultores o coordinadores de propuestas en lo público. Del proceso de normalización contable se afirma que "[...] se encuentra mucho menos desarrollado en el ámbito público que en el privado. Muestra de ello es la mayoría de los organismos normalizadores internacionales, públicos o profesionales, internacionales o regionales, que desarrollan su labor en el ámbito privado" (Torres
Carlón, 2015, p. 75). Para el caso de Colombia, su sistema normativo debió ser reglamentado mediante decretos reglamentarios emitidos por ministerios; tal vez un razonamiento práctico sea que así se daría mayor garantía y respeto al proceso de preparación, transición e implementación por parte de las profesionales, empresas y otras instituciones del estado.

Por otra parte, la idea de un modelo impositivo para un modelo contable, reglamentado y regulado por expedición de normas como son las leyes, los decretos y las reglamentaciones desde el Estado, con el apoyo de profesionales u organismos colegiados y sin el apoyo de las organizaciones del sector real resulta una "idea nefasta" para varios autores, según comenta Assum (1977, p. 150) reflexionando acerca de si la solución en la normalización contable no sería un organismo mixto más amplio donde se incluyan los sectores interesados.

Una idea que se propone por parte de varios autores, entre ellos Mejía et al. (2008) y Senés García (2016), es la presión por otros sectores interesados en los organismos de normalización contable, como son las empresas y organizaciones del sector real o el mismo gobierno, para la aplicación de sus políticas y objetivos económicos y sociales. Mejía et al. (2008) lo proponen desde una posición de reflexión teórica de un modelo contable común para pymes en Latinoamérica y Senés García (2016) sobre una descripción del origen de la normalización contable comparando dos países: Estados Unidos y España. 
De lo expresado anteriormente, Franco (2015) ha denominado el tema como la regulación con interferencia, donde expresa:

[...] en la regulación intervienen diferentes factores entre los que se destacan instituciones, marcos políticos, organizaciones, agentes sociales y procesos de elección; tal complejidad amerita unas reflexiones iniciales sobre el concepto de regulación en general y de regulación contable en particular. (p. 178)

Para esto, se propone que organismos normalizadores contables como el CTCP no son organismos independientes, sino que tienen influencia por parte de otros actores que son mencionados por Mejía et al. (2008) y Franco (2015) como las empresas, los gremios, los agentes sociales y el mismo gobierno, además de las corrientes internas ideológicas frente a un tema de los consejeros, situación que es posible que se vea reflejada dentro del contenido de los conceptos.

\section{Normalización contable}

Sobre la normalización contable se encuentra que "[...] es un proceso que busca métodos y prácticas contables uniformes para la elaboración de la información contable de las empresas, a nivel nacional, facilitando su análisis y comparación" (Gracia-Sarubbi et al., 2014, p. 399); también se establece a la normalización contable como el proceso reglado y organizado que, mediante la elaboración y emisión de normas contables, tiende a la adopción de métodos y prácticas contables homogéneas para las empresas nacionales e internacionales en la elaboración de la información financiera, lo que permite que esta sea comparable y comprensible para los usuarios en cualquier ámbito geográfico (Yubero, s.f., parr. 1).

La normalización contable no es un concepto nuevo a pesar de estar inmerso dentro del movimiento económico y político denominado globalización; Vela Bargues (2004) afirma que:

[...] la normalización contable es tan antigua como la partida doble, en la medida que esta última se ocupa de elaborar normas uniformes para el registro de hechos económicos. Junto a este concepto genérico de normalización, surge, con el paso del tiempo, una segunda acepción, referida ya al fenómeno regulador, con carácter de actividad institucionalizada y organizada, de modo que, con esta óptica, el termino normalización puede considerarse sinónimo de regulación. (p. 310)

De nuevo, con estos conceptos se retoma la institución o institucionalidad para dar garantía y respaldo a la ejecución de procesos como la adopción, la convergencia, la adaptación o la homogeneización de normas contables, situación que puede complementarse con lo expuesto por Yubero (s.f.), quien menciona que "el objetivo de la normalización contable es aumentar la eficacia de la contabilidad como instrumento básico para lograr que los documentos contables sean un eficaz medio de información en las 
relaciones financieras entre las empresas y los usuarios".

Sin embargo, no debe olvidarse que la normalización contable tiene un objetivo y es el de estandarizar el modelo contable para el cumplimiento de los principios contables que logren que la información de las organizaciones satisfaga a los usuarios interesados en ella y así tomar decisiones o contribuir al control interno social, económico y ambiental (García \& Sierra-González, 2010; Ferrer, 2013; Mantilla, 2001; Rueda-Delgado, 2002; Sierra-González, 2008; Assum, 1977).

\section{Concepto (documento)}

El CTCP, como entidad pública y organismo normalizador, utiliza el formato de concepto para resolver las consultas a las personas naturales o jurídicas, las cuales se resuelven de acuerdo con la naturaleza y competencia del tema. El concepto puede definirse como "la conclusión sobre un tema de carácter particular o general, a la que se llega después de un análisis de los hechos y de la normatividad, jurisprudencia y doctrina vigentes y aplicables al tema en estudio"(Secretaria de Gobierno de Bogotá, 2017).

Como se expresa en la definición, el concepto tiene su origen en la consulta que realizan al CTCP para resolver dudas en diferentes temas de la profesión y la disciplina. De acuerdo con nuestra investigación, se establecieron categorías relacionadas con los elementos de los estados financieros, normas contables, políticas y presentación de estados financieros, así como la práctica profesional del contador público.

\section{Materiales y métodos}

El proyecto de investigación se realiza utilizando un enfoque mixto; sin embargo, para este avance, se estableció un enfoque cuantitativo, empleando la estadística descriptiva, orientado por una investigación de tipo documental. Las fuentes de información primarias son conceptos emitidos por el CTCP. Se utilizó una técnica de análisis de contenido, donde los grupos de autores observaron uno a uno los conceptos para clasificar de acuerdo con las variables establecidas. Los datos fueron procesados con el programa Excel usando las funciones de estadística descriptiva. Para este artículo, se utilizó la frecuencia relativa de cada variable para analizar los conceptos desde tendencias, relacionando y contrastando las mismas variables.

El instrumento utilizado para la recolección y organización de la información es una base de datos en Excel denominada matriz de categorización, donde se relacionaron 7191 conceptos emitidos por el CTCP encontrados en la plataforma de la página oficial del CTCP en el período de 1992 a 2018, de los cuales se establecieron variables para categorizar tales como: año de consulta, tema, tipo de persona que realiza la consulta, elementos de los estados financieros y práctica contable.

\section{Resultados}

Lo primero que se realizó en esta etapa fue la revisión del formato que 
presentan los conceptos emitidos. Como se estableció, las fuentes de información principal fueron los 7191 conceptos del CTCP, como documento emitido por una entidad pública, que tiene un formato donde se dan las respuestas a las inquietudes y consultas que son realizadas y radicadas en este organismo. El formato ha tenido una evolución y se ajustó a partir del año 2009. Especialmente, es a partir de 2010 cuando se refleja un cambio en la estructura del formato. Las partes identificadas en el formato son: membrete, radicado, datos del consultor, referencia, consulta, respuesta, responsabilidad del concepto y firma del consejero responsable.

Como se observa en la figura 1, se identifica que el formato mantenía, del año 1992 al 2009, partes básicas: radicado, consulta, respuesta, responsabilidad de la respuesta y firma del responsable. Ya para el año 2010 y en adelante, el formato de los conceptos emitidos se estandariza y mantiene todas las partes mencionadas anteriormente, además de mejorar la presentación y la información presentada.

El segundo paso en el proceso fue depurar los 7191 conceptos publicados y emitidos en la página del CTCP de acuerdo con tres categorías: conceptos publicados, conceptos depurados y conceptos revisados. Los últimos son a los que se les realiza la revisión del contenido, además de constituir la base de la matriz.

La figura 2 muestra que, de los 7191 conceptos publicados por el CTCP, 613, es decir, el 9,31\%, se depuraron por estar anulados dentro de la plataforma o por error al momento de publicarlos o descargarlos. Del total publicado, el 90,68\% es la muestra total que se utilizó para la primera fase general descriptiva.

Figura 1. Partes encontradas en el formato de concepto emitido por el CTCP por año.

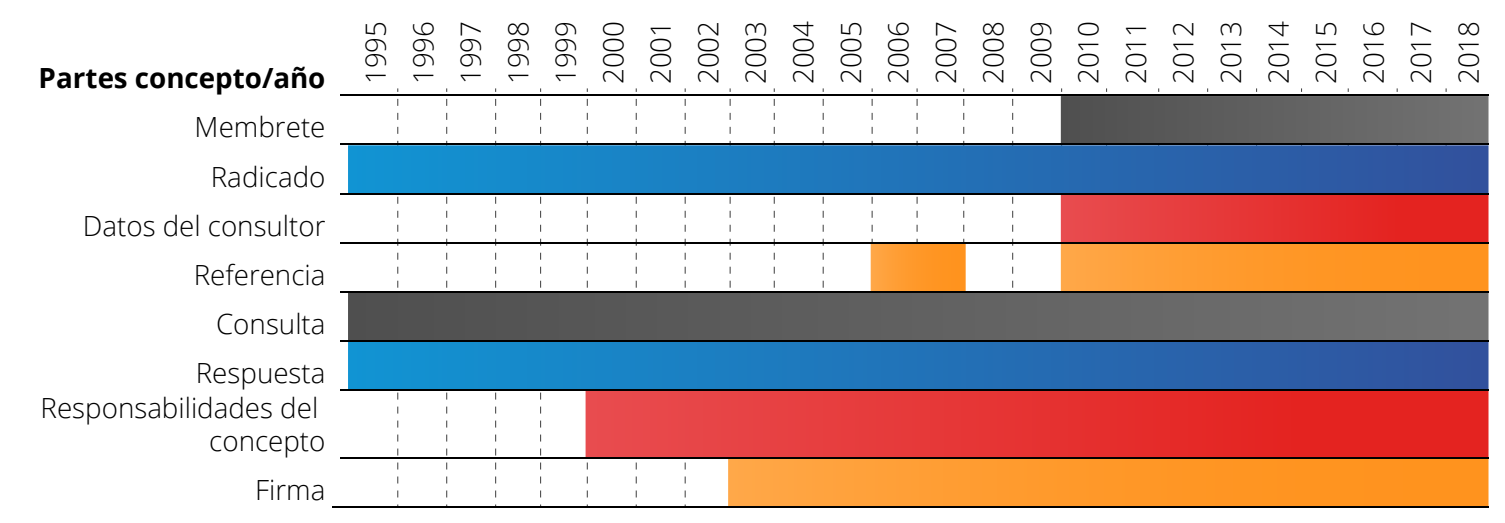

Fuente: información tomada y organizada de la matriz de categorización. Elaborada por los autores. 
Figura 2. Conceptos depurados publicados en la página del CTCP.

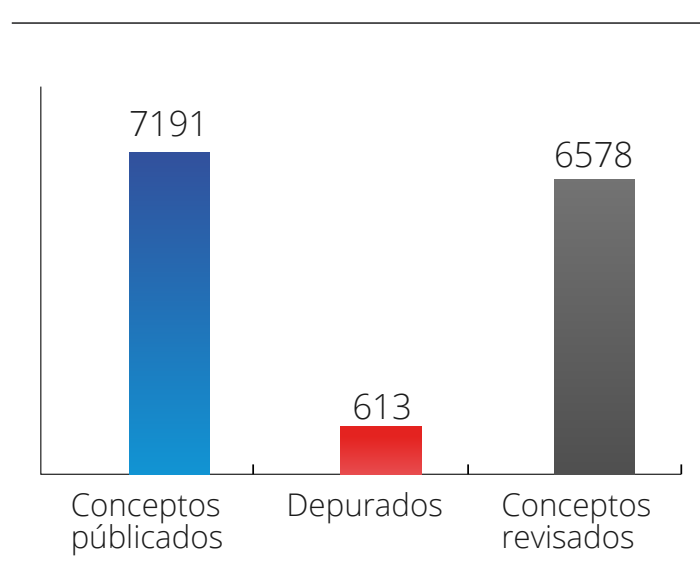

Fuente: información tomada y organizada de la matriz de categorización. Elaborada por los autores.

En este segundo paso, también se pudo encontrar la tendencia de publicación por año de conceptos por parte del CTCP. A continuación, en la tabla 1, se relaciona de acuerdo con su frecuencia.

En la figura 3, se observa mejor la tendencia en el período de 27 años to- mado para la revisión. Del año 1992 al año 2006 se establece la publicación de un número no superior de conceptos publicados de 90. Luego, hay dos picos en el año 2007 y 2010, con 187 y 232 conceptos, respectivamente. En los años 2008 y 2009 se observa un número limitado de conceptos no mayo de 21. A partir del año 2009, la cantidad encontrada de conceptos publicados empieza a subir; sin embargo, es desde el año 2012 que se observa una tendencia creciente de 126 a 1129 conceptos en el año 2018; también se observa que la publicación de conceptos se mantiene a partir de 2015, con un promedio cercano a los 1050 conceptos.

En la figura 4, se muestra el número de conceptos consultados por año de acuerdo con tres categorías: persona natural, persona jurídica y no se identifica. Se relaciona solo el número de conceptos de persona natural para establecer la relación gráfica con las otras dos categorías. Así, se encuen-

Tabla 1.Número conceptos publicados por CTCP por año

\begin{tabular}{c|c|c|c|c|c} 
Año & n.o conceptos & Año & n.o conceptos & Año & n.o conceptos \\
\hline 1992 & 0 & 2000 & 12 & 2009 & 21 \\
\hline 1993 & 0 & 2001 & 50 & 2010 & 232 \\
\hline 1994 & 0 & 2002 & 54 & 2011 & 115 \\
\hline 1995 & 11 & 2003 & 30 & 2012 & 124 \\
\hline 1996 & 34 & 2004 & 21 & 2013 & 401 \\
\hline 1997 & 82 & 2005 & 27 & 2014 & 741 \\
\hline 1998 & 60 & 2006 & 39 & 2015 & 1056 \\
\hline 1999 & 41 & 2007 & 187 & 2016 & 986 \\
\hline & & 2008 & 8 & 2017 & 1114 \\
\hline
\end{tabular}

Fuente: información tomada y organizada de la matriz de categorización. Elaborada por los autores. 
tra que, en el período de 1995 a 2009, los conceptos no identificaban claramente el tipo de persona que solicitaba la consulta; sin embargo, en los que se logró identificar, presentaban una mayor tendencia a ser persona natural. A partir del año 2009, se observa que se mantiene la tendencia alta de consulta por parte de personas naturales y disminuye la tendencia de los conceptos sin identificar por tipo de persona; no obstante, del año 2015 al 2018 se encontró un aumento de tendencia por parte de las consultas en calidad de personas jurí- dicas, categoría que incluye entidades públicas o empresas del estado.

La figura 5 muestra cómo solo a partir del año 2013 se identifica mejor el sector público o privado al cual pertenece la persona natural o jurídica que realiza la consulta. Del año 2013 al año 2017, se observa una consulta mayor o casi igual del sector público en relación con el sector privado; se destacan los años 2017 y 2018, que muestran una alta tendencia de parte del sector privado en la realización de consultas al CTCP.

Figura 3. Tendencia de conceptos publicados por el CTCP en un período de 27 años.

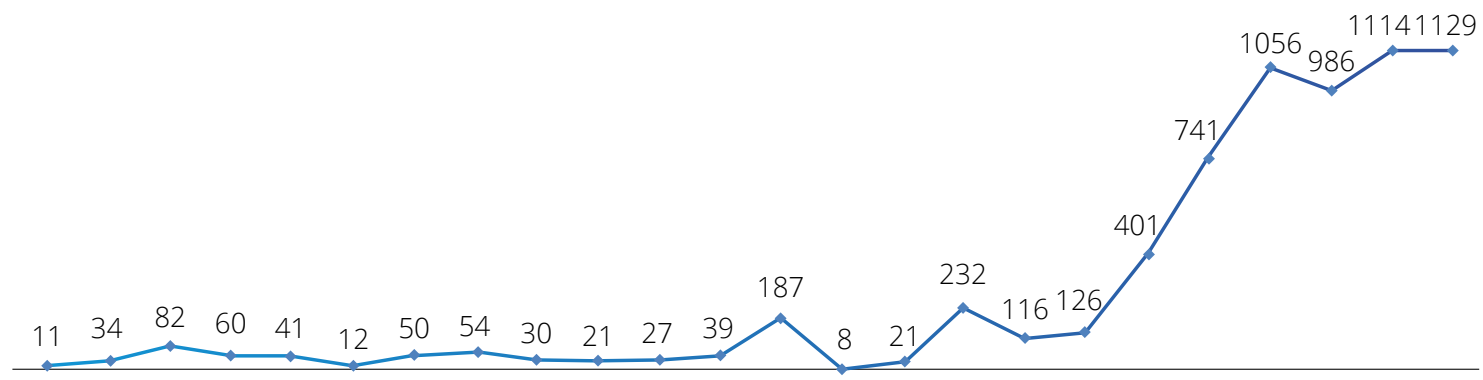

199519961997199819992000200120022003200420052006200720082009201020112012201320142015201620172018

Fuente: información tomada y organizada de la matriz de categorización. Elaborada por los autores.

Figura 4. Tendencia de conceptos publicados por el CTCP en un período de 27 años relacionado con las categorías tipo de persona en la consulta.

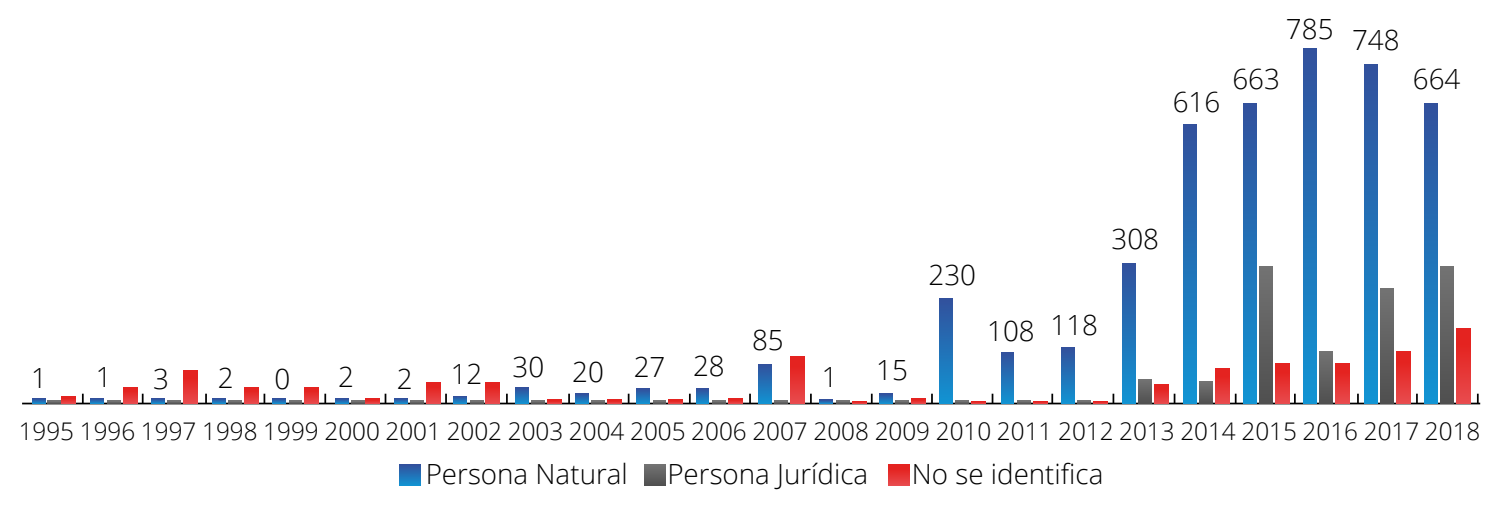

Fuente: información tomada y organizada de la matriz de categorización. Elaborada por los autores. 
Figura 5. Tendencia de conceptos publicados por el CTCP en un período de 27 años relacionado con las categorías sector en la consulta.

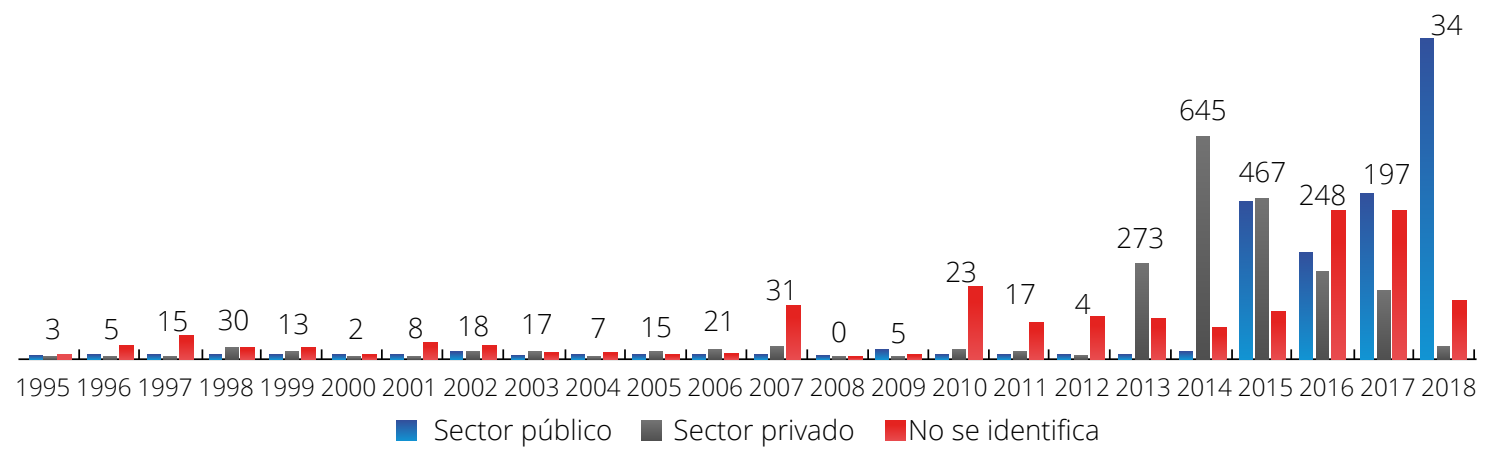

Fuente: información tomada y organizada de la matriz de categorización. Elaborada por los autores.

En la figura 6, se logra observar de manera general y consolidada el tipo de sector de donde provienen las consultas; el sector público y privado muestran una tendencia similar y su número no se diferencia por más de 200 conceptos; sin embargo, el formato de los conceptos publicados por el CTCP no define esta característica, tipo sector, en las consultas, por lo que se encontró un alto número, 2257 conceptos, sin identificar el origen del sector que provienen.

Figura 6. Tendencia del total de conceptos publicados por el CTCP en los 27 años relacionado con las categorías sector de la consulta.

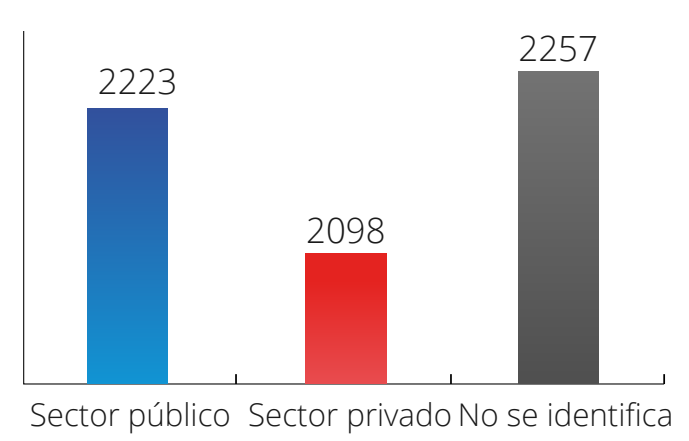

Fuente: información tomada y organizada de la matriz de categorización. Elaborada por los autores.
Como se estableció anteriormente, se relacionaron categorías que se encuentran en el contenido del concepto con elementos de los estados financieros. En la figura 7, se establecen las categorías activo, pasivo y patrimonio relacionadas con el estado de la situación financiera. La principal tendencia que se muestra es las consultas que se enmarcan en el concepto de activos. Gráficamente se puede observar una relación cercana de 2:1 frente a la categoría de pasivos y patrimonio.

En la figura 8, se establecen tres categorías: ingresos, costos y gastos, las cuales se relacionan con el estado de resultados. La categoría de ingresos y gastos presenta mayor tendencia a lo largo de los 27 años revisados en el contenido de los conceptos. La categoría de costos mantiene una relación cercana de 1:2,5 con las otras categorías; sin embargo, es importante recordar que el concepto de gastos y costos tiende a ser confundido y en las consultas se observa, entre otras, las dudas para su clasificación. 
Figura 7. Tendencia de conceptos publicados por el CTCP por año en un período de 27 años relacionado con la categoría elementos del estado de la situación financiera.

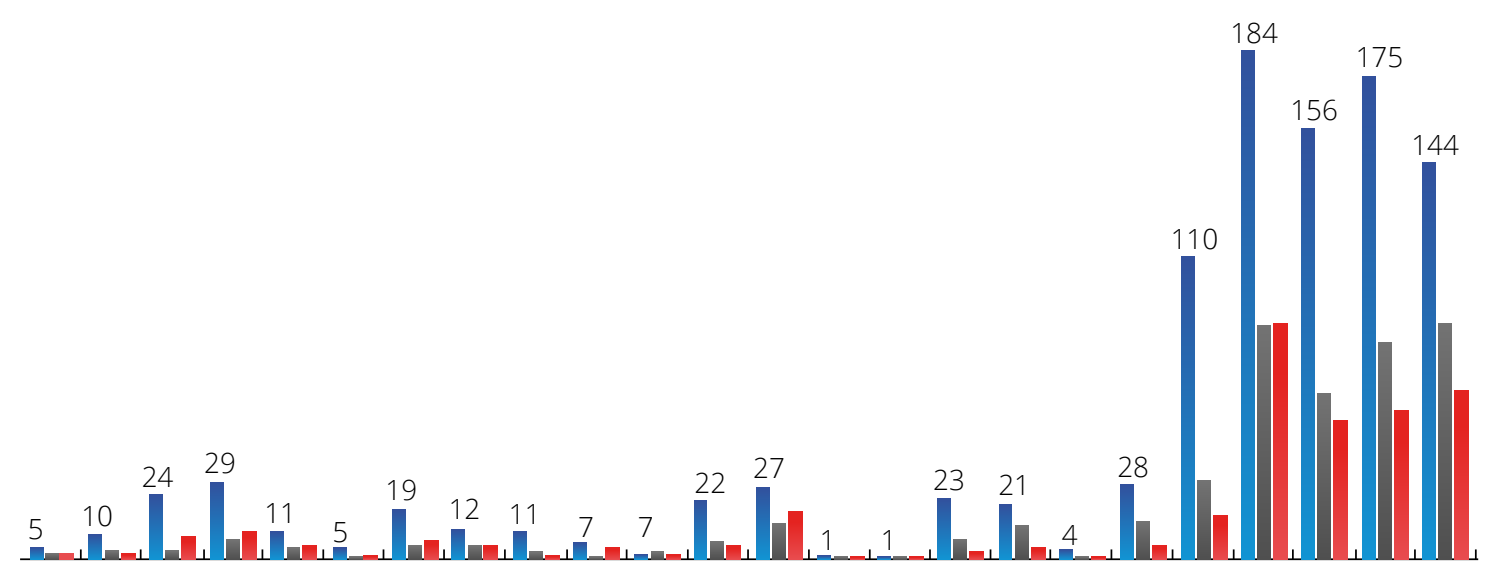

199519961997199819992000200120022003200420052006200720082009201020112012201320142015201620172018

Activo $\square$ Pasivo $\square$ Patrimonio

Fuente: información tomada y organizada de la matriz de categorización. Elaborada por los autores.

Figura 8. Tendencia de conceptos publicados por el CTCP por año en un período de 27 años relacionado con la categoría estado de resultados.

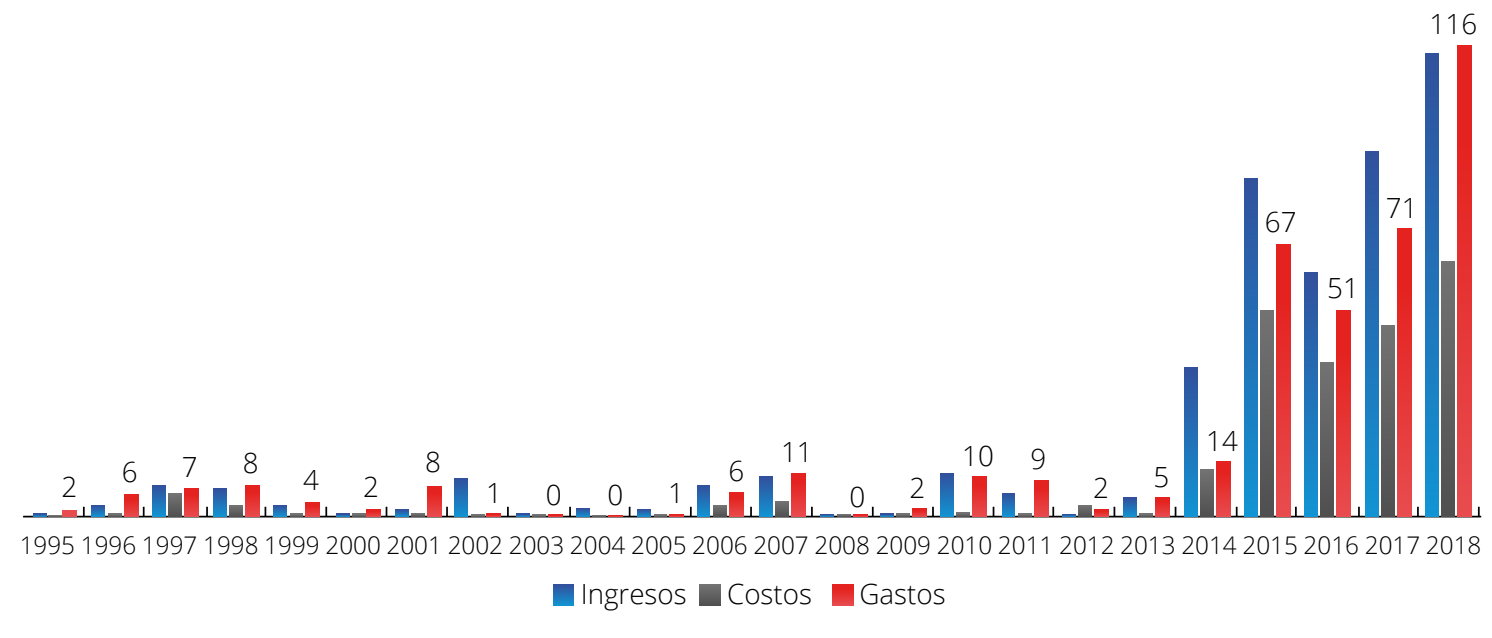

Fuente: información tomada y organizada de la matriz de categorización. Elaborada por los autores.

En la figura 9, se unen las categorías de costos y gastos; esta categoría conceptualmente puede denominarse egresos, así la tendencia de egresos se vuelve 1,5:1 relacionada con la categoría ingresos.
Otras categorías que se definieron fueron: cuentas de orden, estados financieros (donde se incluyeron los temas relacionados con normas y políticas contables) y práctica profesional. En la figura 10 se encuentra que 
la práctica profesional presenta una tendencia alta de consultas al CTCP y mantiene una media cercana de 2,5:1 en relación con la categoría estados financieros; puede verse un aumento de dicha relación en los años 2007 y 2010. Aunque se propone la catego- ría cuentas de orden, se observa poca tendencia en los temas consulta de los conceptos.

De manera general, podemos observar las categorías diseñadas para clasificar los temas de las consultas de

Figura 9. Tendencia de conceptos publicados por el CTCP por año en un período de 27 años, categorías ingresos y costos + gastos (egresos).

Fuente: información tomada y organizada de la matriz de categorización. Elaborada por los autores.

Figura 10. Tendencia de conceptos publicados por el CTCP por año en un período de 27 años relacionado con la categoría otras categorías.

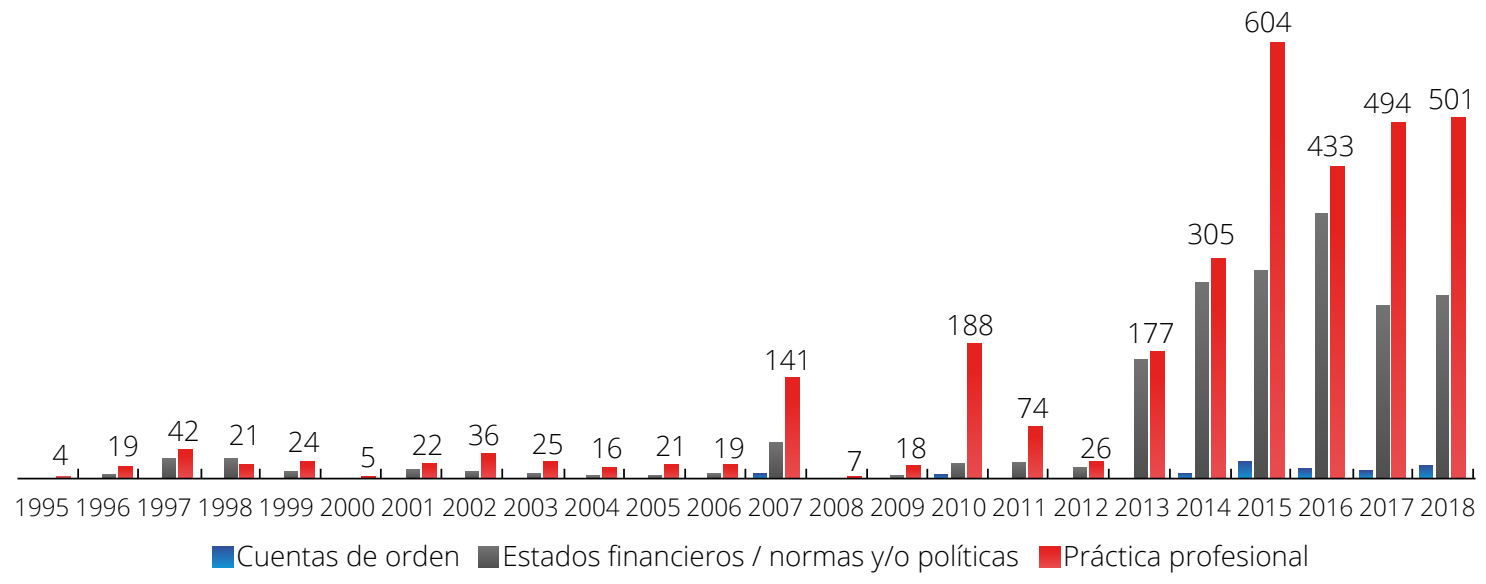

Fuente: información tomada y organizada de la matriz de categorización. Elaborada por los autores. 
los conceptos. En la figura 11, puede establecerse que los que más tendencia presentan en los 27 años son los temas relacionados con la práctica profesional, ya sea como contador, auditor, reviso fiscal u otros temas frente a responsabilidades y ética. Luego le sigue la categoría estados financieros (que relaciona temas con normas y políticas contables), con 1837 conceptos; la categoría activos, con 1036 conceptos, y, como se mencionó anteriormente, la categoría cuentas de orden es la última, con 108 conceptos en los 27 años.

Por último, para los resultados en las figuras 12 y 13, se presentan las nueve categorías relacionadas con el tipo de persona y el sector. Para el primer caso (figura 12), se mantiene la misma tendencia de temas relacionados con la práctica profesional, principalmente las personas naturales, donde se encontraron 2431 conceptos y 522 de personas jurídicas. Continúa la categoría activos, con 705 conceptos para personas naturales y 208 conceptos para personas jurídicas.

Para el segundo caso, la figura 13 presenta la relación entre las nueve categorías y la categoría tipo sector, en la cual se mantiene una tendencia mayor en las categorías práctica profesional, con 1089 en el sector público y 1089 en el sector privado; estados financieros o normas o políticas contables, con 611 en el sector público y 728 en el sector privado; y la categoría activo, con 378 en el sector público y 347 en el sector privado. Las otras categorías mantienen una tendencia similar, especialmente en los conceptos consultados por entidades del sector público, con cerca de 200 conceptos en promedio.

Figura 11. Tendencia de conceptos publicados por el CTCP en un período de 27 años relacionado por categorías.

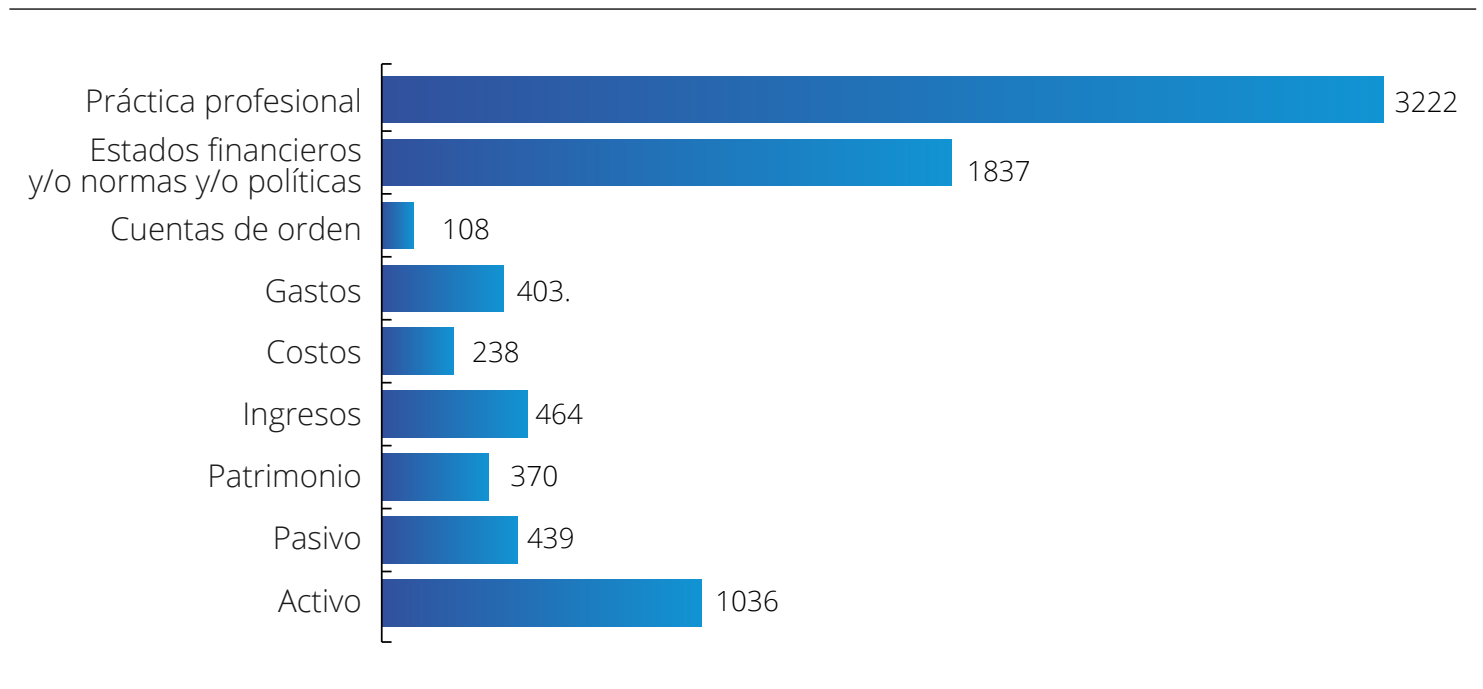

Fuente: información tomada y organizada de la matriz de categorización. Elaborada por los autores. 
Figura 12. Tendencia de conceptos publicados por el CTCP, categoría tipo de persona relacionado.

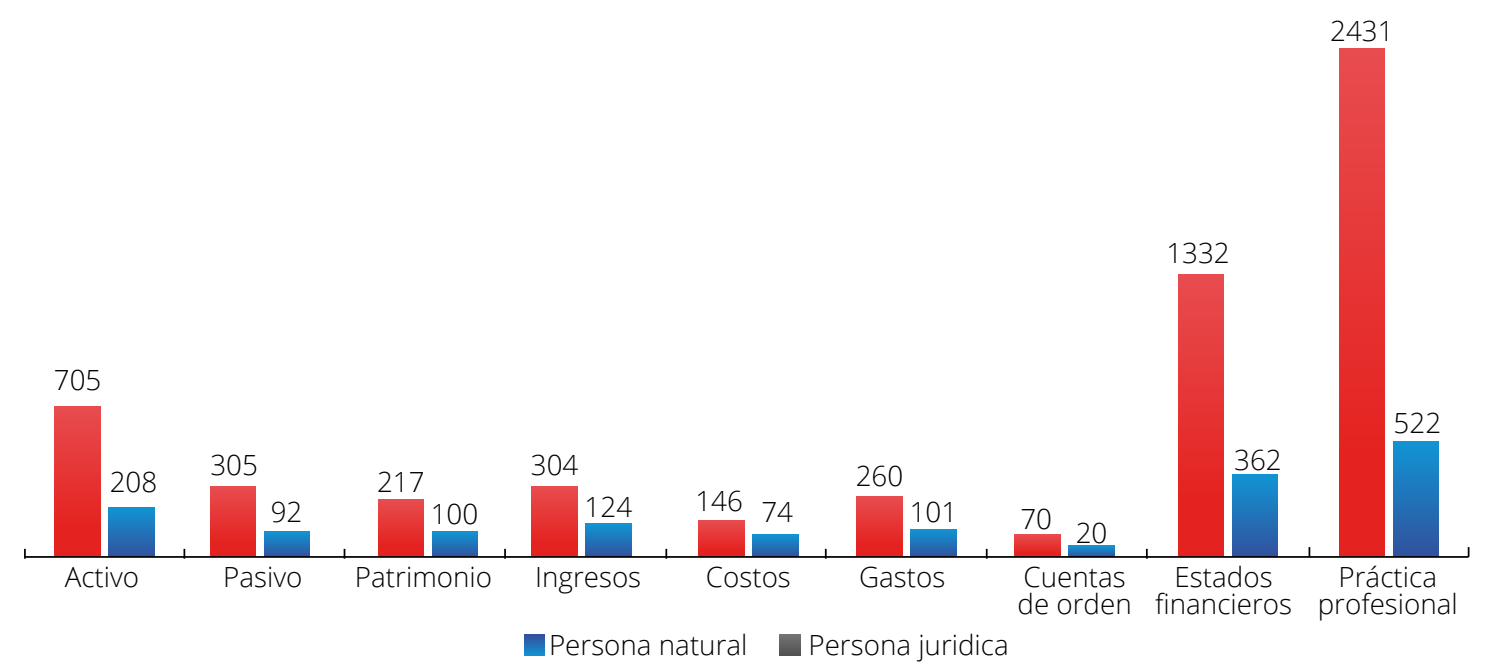

Fuente: información tomada y organizada de la matriz de categorización. Elaborada por los autores.

Figura 13. Tendencia de conceptos publicados por el CTCP, categoría tipo de sector.

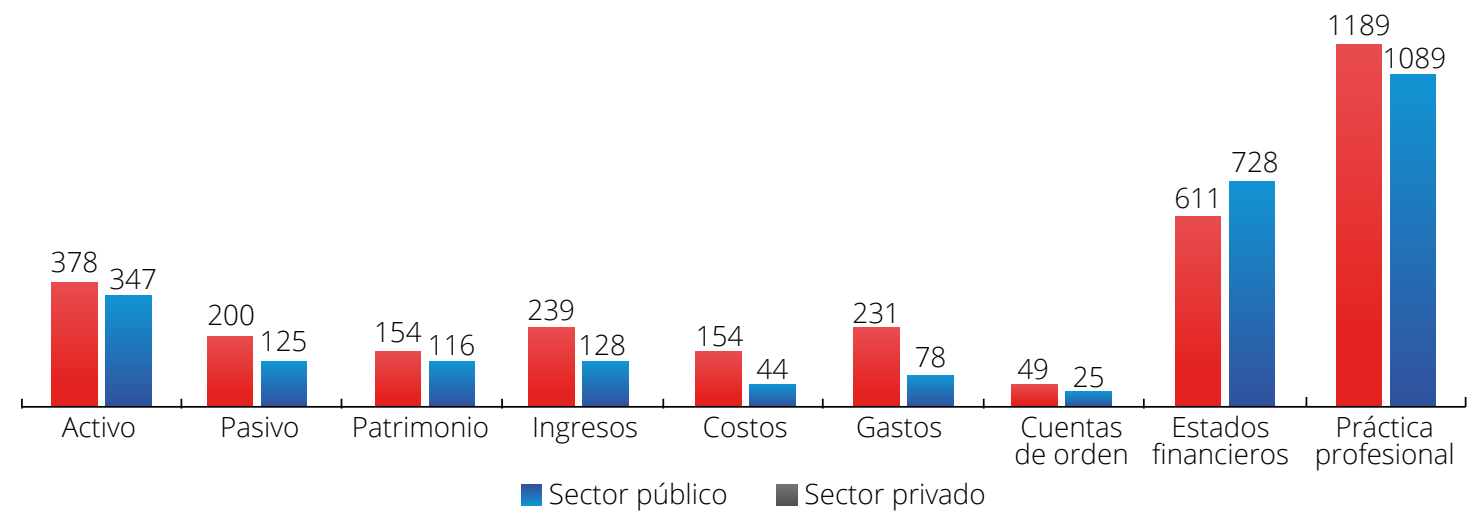

Fuente: información tomada y organizada de la matriz de categorización. Elaborada por los autores.

\section{Discusión}

Los conceptos emitidos y publicados son un aporte del CTCP, como organismo normalizador en Colombia, que ayudan con orientaciones a los usuarios y preparadores de la información en las organizaciones. Lo anterior con el fin de dar claridad sobre aspectos tanto conceptuales como prácticos de las normas contables y profesionales que están vigentes. Como se aprecia en la figura 14, la nube de palabras nos muestra los temas que más se observan en las consultas. Entre estos se encuentran: contador, responsabilidad, contabilidad, contable, dictamen, revisoría, 
tributaria, NIIF, ESFA, clasificación, público, consolidación, traslado, financieros, entre otros temas que suman más de 3006 palabras encontradas en los 6578 conceptos revisados.

Figura 14. Nube de palabras.
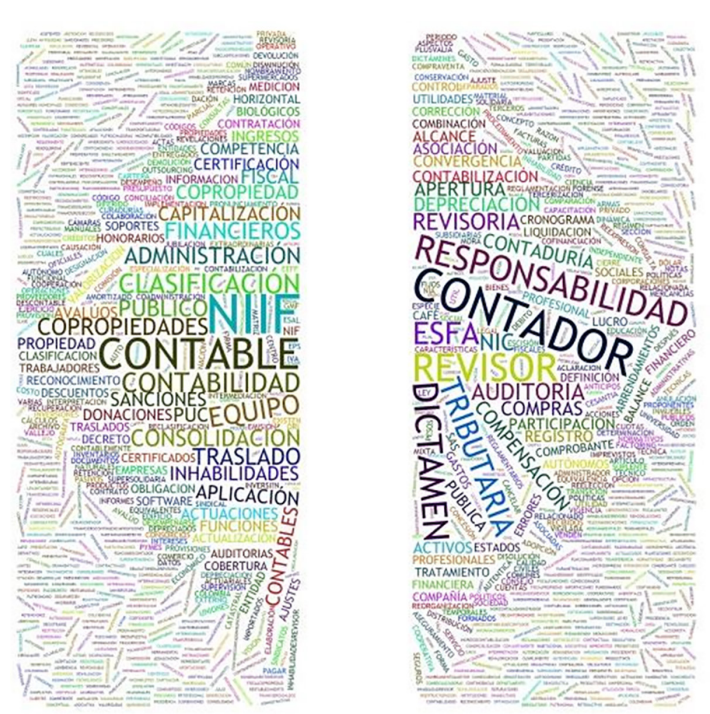

Fuente: elaborada por los autores.

En la nube de palabras se observa que los principales temas tratados en la consulta realizada en los conceptos tienen una relación directa con el CTCP, ya sea con las normas (por ejemplo, los tema de NIIF, ESFA, decreto 2649 y ley 1314), la práctica profesional (con palabras como contador, revisor, auditoria o inhabilidad) o los elementos de los estados financieros (como los activos, los pasivos, el patrimonio, el ingreso y los gastos); sin embargo, es posible ver temas que se relacionan más con el contexto donde se aplican las normas, como las copropiedades, las entidades sin ánimo de lucro o el sector público.

Como lo mencionan Fuentes et al. (2011), el CTCP se convierte en un ente normalizador público, el cual, con la emisión de los conceptos, cumple la función, entre otras, de dar respuesta cuando haya dudas o casos específicos en las consultas o solicitudes de su competencia; sin embargo, es importante apreciar, con base en la figura 3, que esta función se vuelve relevante a partir del año 2009 en adelante, dado que el número de conceptos aumenta de manera lineal hasta el año 2015. Lo anterior es explicable por la primera fase de preparación y transición a las normas internacionales de información financiera y luego, en el período 2015-2018, por la etapa que se da de implementación en los diferentes grupos de empresas.

Lo que se puede preguntar con respecto a la figura 3 es por qué antes del 2009 el número de conceptos emitidos es tan bajo, si la función del CTCP se mantuvo en todo el período trabajado desde 1992, una situación que deja entrever varias suposiciones, como el estancamiento del modelo contable colombiano frente a sus normas, el poco reconocimiento del CTCP como institución y organismo normalizador $y$, al interpretar el aumento a partir de 2009, es posible que sugiera también una poca preparación de los profesionales de la contaduría pública y de las organizaciones privadas y públicas en el tema de normas internacionales de contabilidad. Por tanto, el año 2009 es un año de inflexión y cambio para la contabilidad nacional, sus profesionales y su institución normalizadora.

Con los cambios que surgen a partir del año 2009, el CTCP, como institu- 
ción para el sistema contable colombiano y organismo normalizador, se convierte en un actor principal y de consulta no solo para los profesionales como personas naturales, sino también para personas jurídicas (véase la figura 4) en los dos sectores: público y privado (véase la figura 5). Aunque el sector público tiene un organismo propio, la relación de empresas mixtas en su patrimonio precisa generar consultas en el momento de la aplicación de las NIIF como modelo contable privado, situación que puede verse en la figura 6 , donde se muestra una tendencia similar entre consultas del sector público y privado.

Al revisar las tendencias temáticas de las consultas de los conceptos emitidos y publicados por el CTCP, a lo largo de los 27 años se mantiene una relevancia hacia la categoría de activos, patrimonio (véase la figura 7), ingresos, gastos (véase la figura 8) y práctica profesional (véase la figura 9), lo que propone la preocupación por parte de los consultantes en establecer orientaciones en situaciones relacionadas principalmente con estas categorías. Las consultas dejaron ver situaciones muy específicas de las organizaciones y una mala interpretación de las normas, en especial la confusión de los profesionales en la aplicación de los nuevos marcos normativos.

Lo anterior también es posible verlo con el aumento de las consultas de los conceptos relacionados con la categoría de estados financieros, normas y políticas contables. Se podría proponer que, antes del 2013, el profesional y las organizaciones se preo- cupaban más por el registro que por la presentación de la información en los estados financieros, así como por la construcción de políticas contables (véase la figura 10).

En las figuras 12 y 13, las tendencias de las categorías presentadas contra el tipo de persona y el sector al cual pertenece muestran números de conceptos similares, que no se alejan de lo anteriormente expuesto sobre los temas de mayor consulta, como son los activos, la práctica profesional y lo relacionado con los estados financieros.

Sin embargo, aunque es claro que el CTCP cumple con una de sus funciones, la cual es resolver consultas de su competencia sobre la aplicación de las NIIF, y que posterior al año 2009 es más reconocida como institución, de acuerdo con el gran aumento de número de conceptos que emite y publica, se plantea si el CTCP también se convirtió en un organismo que interpreta y emite, dentro de los conceptos, orientaciones propias asumiendo responsabilidades del organismo emisor y de interpretación principal, como lo es el IASB. Lo anterior podría ir en contravía a lo propuesto por Martínez (2011), que menciona que estos organismos promueven la armonización y ayudan a implantar el modelo adoptado por Colombia; asimismo, puede ocurrir una "[...] 'adopción traslapada', en tanto no se considere el fortalecimiento de un organismo de normalización técnica con poder de negociación que además tome en cuenta [...] los principios de conveniencia nacional, la pro- 
tección del interés público y del bien común" (Bustamante, 2010, p. 94).

Sin embargo, con las respuestas a las consultas el CTCP se establece como una institución que da garantía a la normalización contable en los tres modelos que se crearon a partir de la expedición de la ley 1314, situación que confirma lo expuesto por Sierra (2002) al establecer la importancia de las instituciones consultivas que ayudan a la comprensión regulativa, para este caso normatividad, de carácter privado en un sistema público.

De acuerdo con las tendencias establecidas en los resultados y el aumento del número de conceptos a partir del año 2009, es posible reconocer que el proceso de normalización dentro del país se está consolidando, dado que las consultas se orientan hacia el modelo contable adoptado, situación que se acerca a los expuesto por Gracia-Sarubbi et al. (2014, p. 399) y Yubero (s. f.) al referirse al concepto de normalización contable.

\section{Conclusiones}

Con los conceptos emitidos y publicados por el CTCP en el período comprendido entre 1992 y 2018, se observa la activación de este como organismo normalizador técnico contable a partir del 2009, relacionado directamente con la función que tiene de resolver consultas tanto de personas naturales como jurídicas, ya sea del sector privado o público. Una causa puede ser la expedición de la ley 1314 de 2009, que originó dudas en las etapas de preparación, transición e implementación del modelo NIIF adoptado en Colombia.

En el transcurso de estos 27 años, se encontró que las tendencias principales de temas están relacionadas con los activos, los costos, los gastos y la práctica profesional del contador público. Posterior al año 2009, se vuelven relevantes los temas que incluyen temáticas sobre los estados financieros, las normas y políticas contables. Estas tendencias de temas tienen relación con la competencia del CTCP como organismo normalizador técnico contable; sin embargo, se puede decir que, antes del año 2009, las consultas de los conceptos estaban más orientadas a la parte práctica y técnica; luego del año 2009, a la comprensión de las normas, su aplicación y su objetivo principal, que es la utilidad de la toma de decisiones sobre la información financiera.

Por último, el aumento de las consultas de los conceptos relacionados con los nuevos marcos normativos contables en Colombia propone una interrogante: con el análisis que se realiza a casos particulares de las consultas ¿puede estar generándose una interpretación alternativa a la expuesta por el organismo oficial IASB? Esta situación debe reflexionarse, dado que el modelo ya ha venido presentando algún tipo de excepciones y aceptación de prácticas propias en el país, lo que iría desgastando una de las justificaciones principales, como lo es que se estandarice el modelo contable de acuerdo con el modelo globalizado propuesto y practicado por un gran número de países. 


\section{Referencias}

Almeida Espinosa, A., \& Rojas Prada, G. (2018). Reforma tributaria y el impacto sobre la renta de las personas jurídicas en Colombia. Revista GEON (Gestión, Organizaciones Y Negocios), 5(2), 68-81. https://doi. org/10.22579/23463910.5

Arango, D. \& Torres, A. (2014). Análisis del proceso de armonización de las normas internacionales de contabilidad e información financiera (NIIF-NICSP) en el sector público en Colombia. Revista Contexto, 3(1), 132-145. https://bit. ly/3iPplwT

Assum, J. (1977). Lineamientos para una Introducción a los Llamados Principios de Contabilidad Generalmente Aceptados. Revista de Economía y Estadística, 21(1-4), 119-189. https://bit. ly/3wjuQHU

Bustamante, H. (2010). El interés público en la nueva base institucional de la regulación contable en Colombia. Comentarios generales a propósito del proceso de convergencia hacia estándares contables internacionales. Contaduría Universidad de Antioquia, (56), 81-104. https://bit.ly/3vdaGhv

Franco, R. (2015). Dimensión heterotopológica de la regulación contable. Perspectivas críticas sobre gobierno corporativo y regulación. Revista Científica General José María Córdova, 13(16), 173-199. https://bit.ly/3gvDzzY

Ferrer, A. (2013). Análisis del proceso de convergencia a Normas Internacionales de Contabilidad e Información Financiera desde los factores intrínsecos al sistema contable en Colombia. Cuadernos de Contabilidad, 14(36), 971-1007. https://bit.ly/2ROu5HL
Fuentes, H., Castiblanco, Y. \& Ramírez, M. (2011). Formulación de las NIF en México: referente de convergencia a NIIF. Cuadernos de Administración, 27(45), 11-30. https://bit.ly/3wkPfw3

García, N. \& Sierra-González, E. (2010). Un análisis de la reforma contable en Colombia. Revista Internacional Legis de Contabilidad y Auditoría, 51, 39-54. https://bit.ly/3zpcmal

Gracia-Sarubbi, F., San Juan-Pajares, C. \& Rodríguez-López, A. (2014). Evolución del proceso de armonización contable en España y Brasil en el período 1973-2013. Cuadernos de Contabilidad, 15(38), 397-425. https://doi. org/10.11144/Javeriana.cc15-38.epac

Mantilla, S. (2001). Adopción de estándares internacionales de contabilidad. Revista Legis del Contador, 6(abril-junio), 103-140.

Martínez, G. (2000). Imperio transnacional: transplante y adopción de normas y modelos contables. Contaduría Universidad de Antioquia, (36), 134-147. https://bit.ly/3xhHjfj

Martínez, G. (2011). El empiro-pragmatismo: vector conceptual la regulación contable. Revista FACCEA, 1(1), 20-26. https://bit.ly/2SmZMII

Mejía, E., Montes, C. \& Montilla, O. (2008). Fundamentos teóricos del modelo contable común para las PYMES de América Latina: una alternativa a la regulación contable internacional IASB. Estudios Gerenciales, 24(107), 59-86. https://doi.org/10.1016/S01235923(08)70037-8

Mila, F. \& Yánez, K. (2020). Sistemas de derecho, fuentes y pluralismo jurídico. Horizonte de la Ciencia, 10(19), 74-90. https://doi.org/10.26490/uncp.horizonteciencia.2020.19.589 
Ministerio de Comercio, Industria y Turismo. (2013, 22 de abril). Acuerdo número 1. Por el cual se adopta el reglamento interno de Funcionamiento del Consejo Técnico de la Contaduría Pública. https://bit.ly/3qlEk31

Rueda-Delgado, G. (2002). Descripción de las diferencias de la contabilidad financiera colombiana que pueden dificultar la plena convergencia con los estándares internacionales. Cuadernos de Contabilidad, 4(19), 53-66.

Secretaria de Gobierno de Bogotá. (2017, 3 de febrero). Glosario: Definición de Concepto jurídico. https://bit.ly/3gdPzap

Senés García, B. (2002). El origen de la normalización contable: EEUU versus España. Contaduría Universidad de Antioquia, (40), 19-36. https://bit. ly/3pLCFZf
Sierra-González, E. (2008). Análisis de la relación entre contabilidad y fiscalidad en Colombia. Innovar, 18(31), 117-134. https://bit.ly/3zmDcAt

Sierra, E. (2002). El nuevo periodo de la normalización internacional contable y sus implicaciones en Colombia. Innovar, (19), 89-107. https://bit.ly/3wjA3PY

Yubero, P. (s.f.). Normalización contable. Diccionario económico. Expansión. https://bit.ly/3gi1jlX

Torres Carlón, A. (2015). Capítulo 2. La dimensión internacional de la contabilidad Pública. https://bit.ly/3cCEX2H

Vela Bargues, J. (1994). Contabilidad pública y normalización contable: Una especial referencia al caso español. Revista Española de Financiación y Contabilidad, 24(79), 309-334. https://bit. ly/2TSwdPk 